\title{
Same-day antiretroviral treatment (ART) initiation and associated factors among HIV positive people in Northwest Ethiopia: baseline characteristics of prospective cohort
}

Nurilign Abebe Moges ${ }^{1,2^{*}}$ (D), Olubukola Adeponle Adesina ${ }^{3,4}$, Micheal A. Okunlola ${ }^{3,4}$ and Yemane Berhane ${ }^{5}$

\begin{abstract}
Background: Despite a well-established fact that same-day or rapid ART initiation after a positive HIV test result is vital for faster viral suppression and for prevention of further sexual transmissions of HIV, there is a paucity of evidence on the uptake of same-day ART initiation among newly HIV diagnosed people in Northwest, Ethiopia.

Methods: A cross-sectional study was conducted between December 1st, 2018 and July 30, 2019. About 759 newly HIV diagnosed adults were recruited from 24 health facilities. Data were collected using interviewer-administered questionnaire. Data were entered using EPI-Data and exported to SPSS and STATA software for further analysis. Bivariate logistic regression was used to select candidate variables at $p$-value less than 0.25 for multivariate logistic regression. Then adjusted odds ratio with 95\% Confidence Interval (Cl) at p-value of less than 0.05 was used to declare the statistical associations between the dependent and independent variables.

Result: Magnitude of same-day ART initiation was 318 (41.90\%) [(95\% Cl, 38.2-45.20\%)]. Factors associated with same-day ART initiation were: Patients resided in West Gojjam Zone were 2.04 times more likely to initiate sameday ART compared to those in Bahir Dar city administration $[A O R=2.04(1.04-3.97)]$, patients in the health centers were 3.06 times more likely to initiate same-day ART initiation compared to those in the hospitals $[A O R=3.06$ (1.90-4.92)] and Patients who were diagnosed their HIV status at the same health facility where they linked for ART were 2.16 times more likely to initiate ART at the same-day of diagnosis [AOR $=2.16(91.24-3.74)]$. Moreover, patients with no opportunistic infection $[A O R=2.08(1.04-4.19)]$ and pregnant women $[A O R=3.97(1.78-8.87)]$ were more likely to initiate ART same-day of diagnosis.

(Continued on next page)
\end{abstract}

\footnotetext{
* Correspondence: nure113@gmail.com

'Department of Public Health, College of Health Sciences, Debre Markos University, Debre Markos, Ethiopia

${ }^{2}$ Pan African University, Life and Earth Sciences Including Health and Agriculture Institute (PAULESI), University of Ibadan, Ibadan, Nigeria Full list of author information is available at the end of the article
}

C C The Author(s). 2020 Open Access This article is licensed under a Creative Commons Attribution 4.0 International License, which permits use, sharing, adaptation, distribution and reproduction in any medium or format, as long as you give appropriate credit to the original author(s) and the source, provide a link to the Creative Commons licence, and indicate if changes were made. The images or other third party material in this article are included in the article's Creative Commons licence, unless indicated otherwise in a credit line to the material. If material is not included in the article's Creative Commons licence and your intended use is not permitted by statutory regulation or exceeds the permitted use, you will need to obtain permission directly from the copyright holder. To view a copy of this licence, visit http://creativecommons.org/licenses/by/4.0/. The Creative Commons Public Domain Dedication waiver (http://creativecommons.org/publicdomain/zero/1.0/) applies to the data made available in this article, unless otherwise stated in a credit line to the data. 


\begin{abstract}
(Continued from previous page)
Conclusions: Same-day ART initiation was low among HIV patients in Ethiopia. Patients attending their treatment at hospitals and those from big city (Bahir Dar) were less likely to initiate same-day ART. Clinical factors such as having opportunistic infections and non-pregnancy status affected the immediate initiation of treatment. HIV positive people who seek care in hospitals and those tested HIV positive from another health facilities in which they did not intend to continue their ART follow-up care need special attention.
\end{abstract}

Keywords: New HIV patients, Same-day treatment initiation, ART, Ethiopia

\section{Background}

The World Health Organization (WHO) defines rapid initiation of antiretroviral therapy (ART) as commencement of highly active antiretroviral therapy (HAART) within 7 days of HIV diagnosis. WHO also strongly recommends ART initiation on the same day as HIV diagnosis, after ensuring the person's willingness and readiness to start ART immediately, unless there are clinical reasons to delay treatment [1]. Ethiopia started implementing the "test and treat' policy in 2017 [2]. Same-day ART initiation may be a key approach in reaching the 2020 Joint United Nations Programme on HIV/AIDS goal of $90 \%$ of all people living with HIV (PLHIV) knowing their status, 90\% of those diagnosed receiving sustained ART, and 90\% of those receiving ART achieving viral suppression. It may also be important for achieving the suggested fourth "90\%" goal: improving health-related quality-of-life in PLHIV [3].

Another benefit of same-day or rapid ART initiation after a positive HIV test result is faster viral suppression and halting further sexual transmission of HIV [4-9]. Rapid ART initiation also decreases HIV related morbidity and mortality [10]. Despite such benefits of rapid ART initiation, delaying the initiation of ART is common in many developing countries [11, 12]. Although there are many benefits of rapid ART initiation, increased LTFU has also been reported in Uganda, Nigeria and South Africa [13-15]. Many reasons have been given for delayed commencement of ART and these include patient's choice, prolonged adjustment periods, transport costs due to distance from health facility, stigma and fear of disclosure. Other factors associated with delayed ART uptake are staff shortages, long waiting times, fear of drug side effects, male sex, younger age and the need to take time off work $[11,16]$. Furthermore, there are factors that have been associated with delay or lack of linkage to care including acquiring HIV through heterosexual contact/injecting drug use, younger age at diagnosis, lower levels of education, feeling well at diagnosis and alternate healing systems $[17,18]$. However, the previous studies were undertaken during the pre-test and treat era that emphasized factors associated with poor linkage to ART service, not same-day ART acceptance. In addition, rapid initiation of ART was defined as ranging from 14 to 180 days $[10,11,19-$ 22]. That may not reflect the rapid treatment acceptance as per the new WHO guideline [1].

In South Africa, the same-day initiation of ART was 40.1\% from 2017 to 2018 in which increased from 30.3\% in October 2017 to $54.2 \%$ in June 2018 [15]. In Taiwan, rapid initiation of ART was 33.8\% in 2014 and increasing to $68.3 \%$ in 2017 [8]. Similarly in San Diego, 26 and 48\% of newly diagnosed HIV positive individuals started ART on the same-day of diagnosis and within a week respectively [23]. Though findings from a systematic review favored same-day initiation of ART, compared with standard care [3] it was challenged from healthcare professionals [5], structural factors including HIV testing site $[24,25]$ and from patients side [26-28]. In Ethiopia, about $61 \%$ of the HIV-positive patients were linked to care immediately after testing positive for HIV [29]. However, it does not mean they were started on ART immediately.

The acceptability of the same-day ART initiation among pregnant and lactating women using prevention of mother to child (PMTCT) option $\mathrm{B}+$ is a welladdressed issue in previous studies [25, 30, 31]. Randomized control trials also demonstrated that the same-day ART initiation is feasible and beneficial for patients [32, 33]. However, findings from these scenario may not be generalizable due to the nature of the study subjects and the environment in which the studies were undertaken.

Although many qualitative studies have been conducted about the barriers and facilitators to same-day ART initiation [12, 34-40], there is a paucity of quantitative evidence in this regard. Hence, the current study aimed to address the previous studies' methodological gaps by applying advanced quantitative statistical analysis. Furthermore, same-day ART initiation is affected by patient level, facility level, and community level factors [18]. However, such multi-level factors have not been adequately addressed in previous studies because most used secondary data $[15,18]$.

In Ethiopia, most of the studies that examined factors affecting treatment initiation, late presentation to clinical care, drug adherence and adverse drug reactions used either case-control or retrospective cohort designs [41-46]. These studies used secondary data and were unable to 
adjust for different confounding factors because of incomplete data from routine services. The current study, therefore, overcomes the limitations of previous studies by incorporating primary data collection in the HIV test and treat strategy. Therefore, the study aimed to determine the magnitude of same-day ART initiation among HIV patients and associated factors in the Ethiopian context.

\section{Methods and materials}

\section{Study design}

This study was part of a prospective cohort study to measure both same-day treatment initiation and major clinical outcomes after 6th and 12th months on ART. Newly diagnosed HIV positive individuals were enrolled to the cohort. The study aimed to determine uptake of same-day ART, estimation of time to antiretroviral treatment initiation, determine predictors of timely initiation of ART and determine treatment outcome that include treatment retention and viral suppression at 6th and 12th months of the follow up. The baseline data of the prospective cohort study was used in the current study to determine the magnitude of same-day ART initiation among HIV patients and associated factors in Ethiopian.

\section{Study settings}

The study was conducted between December 1st, 2018 and July 30, 2019, in Gojjam, Northwest Ethiopia. Gojjam has three administrative zones namely, East Gojjam; West Gojjam and Bahir Dar city administration. The administrative centers/towns are Debre Markos, FinoteSelam and Bahir Dar which are located at 300, 364 and $564-\mathrm{km}$ respectively from the capital city of Addis Ababa. According to the 2007 population census conducted by the Central Statistical Agency of Ethiopia (CSA), East Gojjam zone has a total population of 2,153, 937 and West Gojjam Zone including Bahrdar city administration has a total population of 2,106,596 [47].

According to East Gojjam zonal health department report of May 2018, there are 32 ART centers of which 25 are health centers while 7 are hospitals with a total of 16,926 patients. There are 30 ART centers (four hospitals and 26 health centers) in West Gojjam with a total of 9600 patients and similarly, there are 9 ART centers in Bahrdar city administration in which 2 hospitals and 7 health centers with total HIV patients of 5088 December 30, 2019. In all health facilities which are rendering ART service, HIV test and treat program had been started since November 2017. Meanwhile, the Ethiopian national guidelines for comprehensive HIV prevention, care and treatment recommended as a patient should start ART as soon as possible and most should be started in the second visit (after 1 week), while TB patients should initiate ART within two to eight weeks of ant tuberculosis treatment [2].

\section{Participants}

Source populations were all newly diagnosed HIV positive individuals between December 2018 and July 30, 2019, in Gojjam, Northwest Ethiopia. The study population were all adult HIV positive individuals from the selected health facilities in Gojjam, northwest Ethiopia and those who fulfilled the inclusion criteria. All adults who gave consent to participate in the study were included in the study.

Eligibility criteria were, all adults ( $>=18$ years) who were newly diagnosed as HIV positive in the selected health facilities were included in this study. The HIV test can be in the same health facility or referred from other health facilities or community settings. Patients enrolled in treatment 7 days prior to data collection were included in the data collection. While the exclusion criteria were that treatment interruption and re-start as "new" (for example as in the case of mothers in the previous PMTCT guideline before option $\mathrm{B}+$ ), health facilities with less than five new patients per month were excluded to use the available resources effectively.

The sample size was determined using both a single population formula for the first objective (prevalence of same-day ART acceptance) and double population formula for second objective (the associated factors) then the highest sample size was considered. Prevalence of same-day ART acceptance (54.2\%) was used from South African study [15]. Accordingly, the sample size using single population proportion formula was 381 study participants were required. By adding a $10 \%$ attrition rate, it was 421 . For the second objective, double population proportion formula was used and assumptions like $80 \%$ power, 95\% confidence interval and 5\% non-response rate were considered. Proportions used in this formula were from primary and secondary clinical outcomes $(\mathrm{P} 1=66.10 \%$ exposed/delayed ART initiation) and $(\mathrm{P} 2=$ 56.21 non exposed/early initiation of ART) from a previous study [33] the largest sample size was 844 .

\section{Variables of the study}

The dependent variable was same-day ART initiation with categorical response of (Yes, No) while the independent variables were grouped into two. Namely individual level and community or health facility level variables. From the individual level variables, sociodemographic characteristics such as age, sex, religion, ethnicity, marital status, educational status, occupation, wealth status, living arrangement, have child/children and family size. Baseline clinical characteristics which included opportunistic infections, body mass index (BMI), functional status, pregnancy status, any current health complaints, WHO clinical stage and mode of HIV testing (VCT or PICT). Behavioral characteristics were serostatus of sexual partner, disclosure status, and HIV test 
history, number of sexual partner/s, condom utilization, and alcohol use. HIV and ART related Knowledge, history of STI. Psychological characteristics were identified via perceived stigma and psychological distress. In addition to this, we documented dates of HIV test confirmed, enrolment to HIV care and ART start. Community and health facility level variables were patient residence (rural, urban), zones (East Gojjam, West Gojjam and Bahir Dar City Administration), health facility type (health center or hospital), and distance from health facility, tested in the same facility and living within the catchment area.

\section{Data collection instruments}

In this study, structured questionnaires were used to collect information during the enrolment period. Data collection tool was developed by reviewing similar literature $[18,21,22,41,48-53]$ and from Ethiopian HIV/ART care intake form, ART/HIV care follow up form and ART cohort registrations [2]. Psychological distress tool was adopted from Kessler-10 scale [54] which has been validated in Ethiopia [43].

\section{Sampling technique}

In the three administrative zones, there are 72 public health facilities rendering ART service. We took 3 months of performance reports of all health facilities to estimate an adequate sample size given the available time and resources. From a total of 24 health facilities, based on their monthly average patient flow and using the previous three-month report, we estimated 8 months data collection period. We divided the total sample size over the health facilities proportionate to the patient flow. A consecutive sampling technique was used to access study participants. Hence we included 24 health facilities with an average case flow of three to five new cases per month (11 from East Gojjam, 8 from west Gojjam and 5 from Bahir Dar (see Fig. 1).

Data were collected on the same-day of HIV diagnosis or in the next visit (within 7 days). Research assistants were nurses working in the ART clinic. A consecutive sampling technique was used to access the study participants. Patient exit interview was conducted in a quiet separate room.

\section{Data quality assurance}

Research assistants were nurses who have received training on the Ethiopian HIV/ART guideline and working in the ART clinic. Two days of training was given about the objectives of the research, methods of data collection, patient right, informed consent, confidentiality and client approach. To make the training concept uniform and to be cost-effective all recruited research assistants were invited to a central place for the training. The training also involved a field practicum in ART clinics. Pretest of the data collection tools was conducted among $5 \%$ of the sample in Wuseta Health Centre (Debre Markos) that was not included in the final data collection. Concept clarity, logical order and reliability was tested. The time required to undertake the interview was also judged and necessary adjustments were made. To minimize social desirability bias, we have employed patient exit interview in a quiet separate room and discussed the objectives of the study. However, some patients were not ready due to the "emergency" nature of learning their HIV status. In this case, we allowed the patients to absorb the meanings of test result for additional few days before interview.

\section{Data processing and analysis}

Data was entered into EPI-Data version 3.5 and were exported to SPSS version 24 and STATA version 14 for further analysis. Data were cleaned and descriptive analysis carried out using SPSS version 24. Bivariate and multivariate logistic regression analysis were carried out using STATA version 14. The Hosmer and Lemeshow's goodness-of-fit statistic was computed and a larger $p$ value (greater than 0.05) was considered for further analysis. We have also used receiver operating characteristics curves (ROC) to measure the discriminatory capacity of the final model. In the bivariate analysis, we have assessed the crude association using each independent variable with the outcome variable (same-day ART initiation) and those variables with $p$-value less than 0.25 included in the multivariate logistic regression model. Finally, a statistical association was declared using adjusted odds ratio (AOR) with 95\% confidence interval (CI) at p-value less than 0.05 .

\section{Operational definitions}

The same-day initiation of ART was defined as acceptance of ART on the same-day of HIV diagnosis and return to home with prescribed medication (ART). Those who answered $>2$ from the five HIV modes of transmission questions were categorized as having good knowledge and $<2$ as poor knowledge [49]. Similarly, those who answered $>2$ from the five modes of HIV prevention questions were considered as having good knowledge and $\leq 2$ as having poor knowledge [49]. Patients' knowledge of ART score above the mean score was considered as knowledgeable. Patient's perceived stigma score above the mean score was considered as high perceived stigma. Psychological distress was measured using Kessler (10); people who score under 20 are likely to be well, score 20-24 are likely to have a mild mental disorder, and score 25-29 are likely to have a moderate mental disorder and score 30 and over are likely to have a severe mental disorder [54]. 


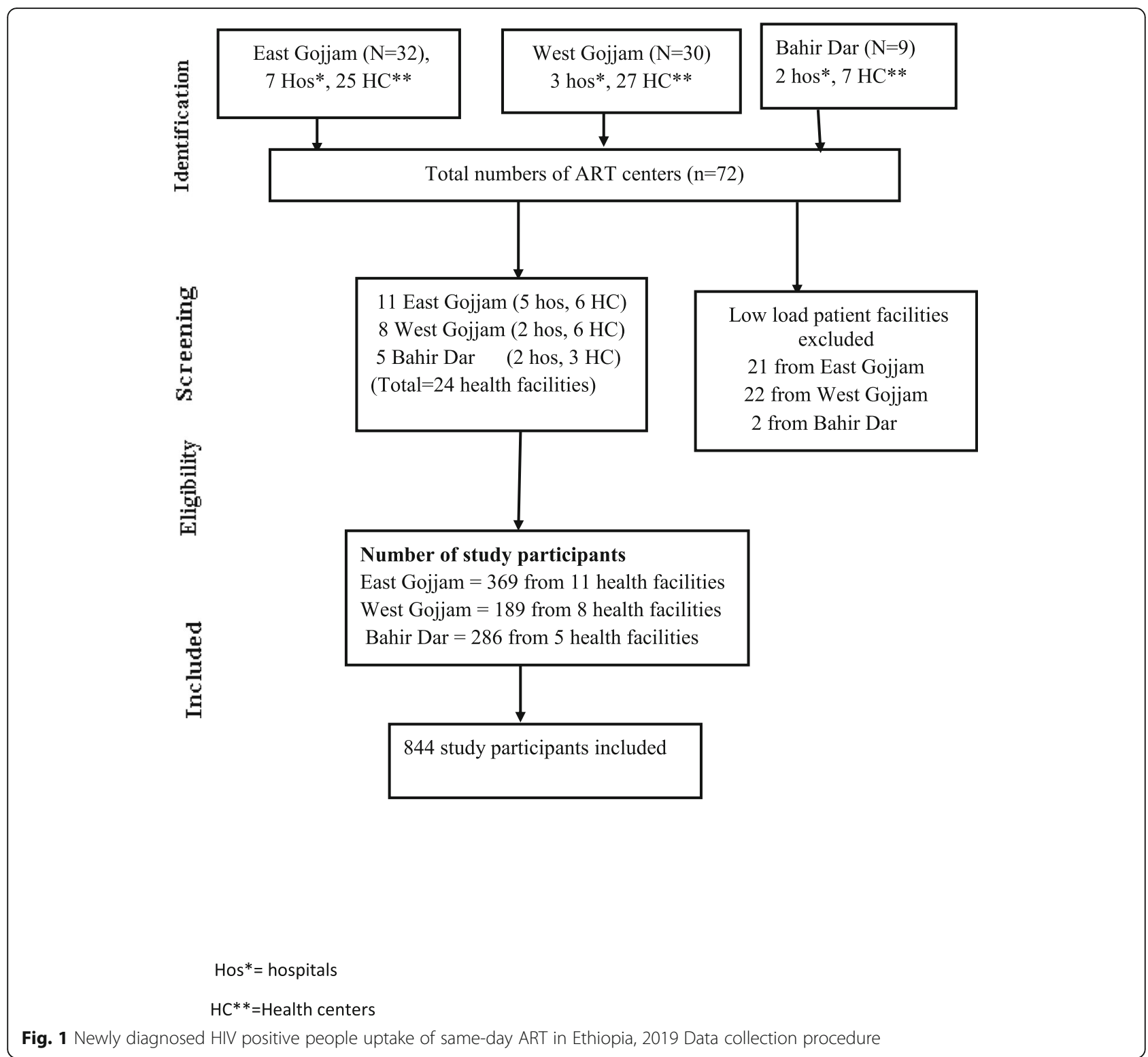

\section{Ethical considerations}

The proposal was approved by the joint University of Ibadan/University College Hospital, Ibadan Institutional Review Board (IRB) of the College of Medicine, University of Ibadan, Nigeria (reference number UI/EC/18/0463). It was also approved by IRB at Debre Markos University (reference number HSC/30/02/2011). We obtained written informed consent from each study subject.

\section{Results}

\section{Socio-demographic characteristics}

A total of 759 participants were included in this study with a response rate of $90 \%$. About 436 (57.40\%) were females. The mean age of study participants was 33.02 with a standard deviation (SD) of \pm 9.16 years. The majority 719 (94.70\%) were Ethiopian Orthodox Christian followers by religion and $747(98.4 \%)$ were Amhara by ethnicity. Four hundred ninety six $(65.30 \%)$ were employed. More than half 397 (52.30\%) had more than one child (Table 1).

\section{Health facility and community-related characteristics of study participants}

About 331 (43.60\%) were recruited from East Gojjam zone and $381(50.20 \%)$ were selected from hospitals. Majority 577 (76\%) were urban residents with 592 (78\%) of them living within less than one-hour walking distance from the health facility. About 584 (76.90\%) were tested HIV in the same facility in which they were linked to ART service while 513 (67.60\%) were diagnosed 
Table 1 Socio-demographic characteristics of newly initiated HIV patients in northwest Ethiopia, 2019

\begin{tabular}{|c|c|}
\hline Characteristics & Frequency (\%) \\
\hline \multicolumn{2}{|l|}{ Sex } \\
\hline Male & $323(42.60)$ \\
\hline Female & $436(57.40)$ \\
\hline \multicolumn{2}{|l|}{ Age in years } \\
\hline $18-24$ & $106(14)$ \\
\hline $25-34$ & $350(46.10)$ \\
\hline$>=35$ & $303(39.90)$ \\
\hline \multicolumn{2}{|l|}{ Marital status } \\
\hline Single & $228(30)$ \\
\hline Married & $278(36.60)$ \\
\hline Divorced & $202(26.60)$ \\
\hline Widow/d & $51(6.70)$ \\
\hline \multicolumn{2}{|l|}{ Religion } \\
\hline Orthodox Christian & 719 (94.70) \\
\hline Muslims & $33(4.30)$ \\
\hline Protestant & $7(0.90)$ \\
\hline \multicolumn{2}{|l|}{ Educational status } \\
\hline No formal education & $282(37.20)$ \\
\hline Primary education & $249(32.80)$ \\
\hline Secondary or higher & $228(30)$ \\
\hline \multicolumn{2}{|l|}{ Employed status } \\
\hline Employed & $496(65.30)$ \\
\hline Not employed & $263(34.70)$ \\
\hline \multicolumn{2}{|l|}{ Occupation } \\
\hline Farmer & $137(18.10)$ \\
\hline Private employed & $89(11.70)$ \\
\hline Merchant/business & $157(20.70)$ \\
\hline Government employee & $101(13.30)$ \\
\hline Daily labor & $170(22.40)$ \\
\hline Student & $24(3.20)$ \\
\hline House wife & $69(9.10)$ \\
\hline Others $^{a}$ & $12(1.60)$ \\
\hline \multicolumn{2}{|l|}{ Have $>1$ child } \\
\hline Yes & 397 (52.30) \\
\hline No & $362(47.70)$ \\
\hline \multicolumn{2}{|l|}{ Wealth quintile status } \\
\hline Poorest & $168(22.13)$ \\
\hline Poorer & $136(17.92)$ \\
\hline Middle & 149 (19.63) \\
\hline Wealthier & $158(20.82)$ \\
\hline Wealthiest & $148(19.50)$ \\
\hline
\end{tabular}

Others $^{\mathrm{a}}=$ (carpenter, handicraft men) following providers initiated testing and counseling (Table 2).

\section{Baseline clinical characteristics}

Three hundred eighteen (41.90\%); (95\% CI, 38.2 to $45.20 \%$ ) were initiated on ART on the same-day of HIV diagnosis. About 550 (72.50\%) had no opportunistic infections at baseline. Majority $505(73.60 \%)$ has BMI greater than 18.5 (Table 3 ).

\section{Behavioral characteristics}

Majority, $572(75.4 \%)$ of patients had disclosed their HIV status to someone. About 745 (98.2\%) had a history of sexual intercourse. Four hundred twenty nine (57.6\%) of the patients have sexual partners with unknown HIV status. About 548 (73.6\%) had never used condoms (Table 4).

\section{HIV/ART related knowledge of respondents}

Patients were asked to list all possible ways of HIV transmission, prevention mechanisms and ART related issues. Six possible answers were determined for ways of HIV transmission while five items were for HIV prevention. We have calculated Cronbach alpha $(\alpha)$ for each knowledge related responses and found $\alpha=0.81$ for HIV transmission questions and $\alpha=0.78$ for HIV prevention questions. Similarly, we have used six-item question to

Table 2 Health facility and community related characteristics of HIV patients in Gojjam, northwest Ethiopia, 2019

\begin{tabular}{ll}
\hline Characteristics & Frequency (\%) \\
\hline Zone & $331(43.60)$ \\
East Gojjam & $170(22.40)$ \\
West Gojjam & $258(34.00)$ \\
Bahir Dar city & \\
Facility type & $378(49.80)$ \\
Health center & $381(50.20)$ \\
Hospital & \\
Residence of the patient & $182(24)$ \\
Rural & $577(76)$ \\
Urban & \\
Distance form health facility & $592(78)$ \\
$<1 \mathrm{~h}$ walking & $167(22)$ \\
$>=1 \mathrm{~h}$ walking & \\
HIV diagnosis made & $584(76.90)$ \\
The same facility & $175(23.10)$ \\
Another facility & \\
Testing modality & $513(67.60)$ \\
Provider initiated & $246(32.40)$ \\
Self-initiated &
\end{tabular}


Table 3 Baseline clinical characteristics of HIV patients in northwest Ethiopia, 2019

\begin{tabular}{ll}
\hline Characteristics & Frequency $(\%)$ \\
\hline Same-day ART initiated & \\
Yes & $318(41.90)$ \\
No & $441(58.10)$
\end{tabular}

ART initiated within seven days of Dx.

$\begin{array}{ll}\text { Yes } & 498(65.60) \\ \text { No } & 261(34.40)\end{array}$

Reason of differing same-day ART $(n=441)$

$$
\begin{aligned}
& \text { Not ready** } \\
& \text { Ol disease }
\end{aligned}
$$$$
383(86.80)
$$

$58(13.20)$

\section{Opportunistic infection $\left(\mathrm{Ol}^{* *}\right)$}

$\begin{array}{ll}\text { Yes } & 209(27.50) \\ \text { No } & 550(72.50) \\ \text { BMI } & \\ <16 & 39(5.70) \\ 16-18.5 & 142(20.70) \\ >18.5 & 505(73.60)\end{array}$

\section{Functional status}

Working

659 (86.82)

Ambulatory/bedridden

100 (13.18)

\section{Symptomatic presentation}

Yes

No

277 (36.50)

$482(63.50)$

WHO clinical stage

I/II

III/IV

Ol prophylaxis within 7 days of diagnosis

Cotrimoxazole only
Isoniazide preventive therapy only
CPT and INH

No prophylaxis

Pregnancy status $(n=436)$

$\begin{array}{ll}\text { Pregnant } & 46(10.61) \\ \text { Not pregnant } & 390(89.40)\end{array}$

Not ready** $=$ (reasons were: need time to discuss with family, competing health priority), $\mathrm{Ol}^{* *}=$ opportunistic infections such as tuberculosis, diarrheal disease, fungal disease and others

discriminate ART related patient knowledge and find $\alpha=0.76$. Cut of points were considered at the mean value of each score of respondent knowledge questions. From a total of the respondent, 477 (62.85\%), 516 (67.98\%) and 478 (62.8\%) have good knowledge about ways of HIV transmission, HIV prevention and ART related knowledge respectively.
Table 4 Behavioral characteristics of HIV patients in Gojjam, northwest Ethiopia, 2019

\begin{tabular}{ll}
\hline Characteristics & Frequency (\%) \\
\hline Disclosed HIV status & \\
Yes & $572(75.4)$ \\
No & $187(24.6)$
\end{tabular}

\section{Living arrangement}

Living with someone

$484(63.8)$

Living alone

$275(36.2)$

Ever had sexual intercourse

Yes

745 (98.2)

No

$14(1.8)$

Partner HIV status $(n=745)$

HIV Positive

$229(30.7)$

HIV negative

$87(11.7)$

Unknown

$429(57.6)$

Life time sexual partner $(n=745)$

One

$305(40.9)$

more than one

$440(59.1)$

Sexually active in the last 3 months

Yes

$398(53.4)$

No

$347(46.6)$

No. of sexual partners last 3 months

One

$356(89.4)$

more than one

$42(10.6)$

Ever use condom $(n=745)$

Yes

$197(26.4)$

No

$548(73.6)$

Frequency of condom use ( $n=197)$

Most of the time

$39(19.8)$

Often

$45(22.8)$

Sometimes

$113(57.4)$

Condom in the most recent sex $(n=197)$

Yes

$103(52.3)$

No

94 (47.7)

Sex out of regular partner $(n=745)$

Yes

161 (21.6)

No

$584(78.4)$

Current STI $(n=745)$

Yes

49 (6.6)

No

696 (93.4)

Ever use alcohol

Yes

546 (71.9)

No

$213(28.1)$

Current use of alcohol

Yes

172 (31.5)

No

374 (68.5) 
Level of psychological distress and perceived stigma Patients' initial level of mental distress was found to be well (371, 41.77\%), mildly distressed (173, 22.79\%), moderately distressed $(136,17.92 \%)$ and severely distressed $(133,17.53 \%)$. The reliability of the tool was $(\alpha=0.90)$. Generally, $371(41.77 \%)$ of patients were well and 442 (58.23\%) were mentally distressed. Similarly, level of perceived HIV related stigma was measured using the tool with a reliability score of $(\alpha=0.61)$ and $599(78.92 \%)$ were found to have a high level of perceived stigma while $160(21.08 \%)$ had a low level of perceived stigma.

\section{Goodness of fit of the model}

We used the receiver operating characteristics curve (ROC) to measure goodness of fit of the model. The area under ROC curve was $76.49 \%$.

Factors associated with uptake of same-day ART initiation In the bivariate logistic regression, the role of several independent variables associated with the outcome variable at $p$-value less than $25 \%$ were analyzed. These included factors from the socio-demographic variables (educational status, occupation and wealth index), factors from the health facility and community-related factors (zone, types of health facility, patient residence, where HIV was diagnosed and HIV test modality) and behavioral related variables (which included having multiple sexual partners, sexually active in the last 3 months, ever use of condom, had sex out of regular sexual partner and ever use of alcohol). Other independent variables included knowledge of HIV prevention methods and level of perceived stigma and, finally clinical related variables (baseline WHO clinical stage, pregnancy status and baseline opportunistic diseases) (Table 5).

In the multivariate logistic regression, about five variables were statistically significant. Patients in West Gojjam Zone were 2.04 times more likely to initiate ART compared to those in Bahir Dar city administration [AOR, 2.04, (95\% CI, 1.04-3.97)]. Similarly, patients in the health center were 3.06 times more likely to accept same-day ART initiation compared to those in the hospital [AOR, 3.06, (95\% CI, 1.90-4.92)]. Patients who had their HIV status diagnosed the same health facility where they were linked for ART were 2.16 times more likely to initiate the same-day of diagnosis. Finally, patients with no opportunistic disease and pregnant women were more likely to initiate ART (Table 5).

\section{Discussion}

This study found that the magnitude of same-day ART initiation was $41.9 \%$ (95\% CI, 38.2-45.20\%) and factors associated with same-day ART initiation were having opportunistic infections, being pregnant, having HIV status diagnosed at the same health facility where one is linked to ART service, accessing care at a health center compared to a hospital and being in West Gojjam administrative zone.

Same-day treatment initiation was low (41.9\%) in the study area. But there was increasing in the proportion of newly diagnosed HIV positive people who initiated ART within 7 days of HIV diagnosis to $65.6 \%$. The finding was in line with a study in South Africa with overall same-day ART initiation of $40.1 \%$ and initiated within 7 days to $62.86 \%$ [15] and similar with study from Taiwan reported $68 \%$ rapid ART initiation within 7 days of HIV confirmed diagnosis in 2017 [8]. However, sameday ART initiation was lower compared to the recent finding of $54.2 \%$ in June 2018 in South Africa [15], Zimbabwe (65\%) [55], 94.9\% in US [56] and San Francisco (96\%) [57]. The high discrepancy observed between the findings of the current study and the previous studies may be due to the patients receiving multidisciplinary evaluation, support, and insurance enrollment/optimization in the San Francisco and US studies. This was not the case in our current study. Additionally, the present study settings included both primary and secondary healthcare facilities, while the study in South Africa was only from primary healthcare facilities. It must be noted that patients who present to primary healthcare facilities are more likely to have uncomplicated presentation.

On the other hand, the magnitude of same-day ART initiation and rapid ART initiation within a week were better than the previous study in San Diego which was 26 and 48\% respectively [23]. The possible reason for the difference observed may be because the former study was undertaken before the WHO official recommendation of immediate ART initiation. Moreover, in a cluster-randomized trial study in rural South Africa it was found that only $36 \%$ initiated ART within a month. This implies that there is an increasing rate of rapid ART initiation after the official declaration of rapid ART following HIV confirmed test by WHO.

Patients without opportunistic infections were two times more likely to initiate same-day ART compared with patients with one or more opportunistic infections. The finding is similar to a study in South Africa which reported that patients who presented with less advanced clinical disease were more likely to accept same-day ART initiation [15]. However, such findings are in contrast with several qualitative studies that had reported the absence of symptoms or signs of ill health, which is the major reason for differing same-day ART [34-36, 58]. Moreover, Ethiopian Ministry of Health and WHO guidelines recommended that a rapid approach to ART initiation is particularly relevant to people with advanced HIV disease $[1,2]$ yet CD4 count determination was not available in the study area during the study period. The discrepancy may a result of healthcare providers who 
Table 5 Factors associated with uptake of same-day ART initiation in northwest Ethiopia, 2019

\begin{tabular}{|c|c|c|c|c|c|}
\hline Characteristics & Same-day initiated & Not initiated & COR $95 \% \mathrm{Cl}$ & AOR $(95 \% \mathrm{Cl})$ & $P$-value \\
\hline \multicolumn{6}{|l|}{ Educational status } \\
\hline No formal education & 126 & 156 & 1 & 1 & \\
\hline Primary education & 121 & 128 & $1.17(0.83-1.65)$ & $0.99(0.56-1.74)$ & 0.987 \\
\hline Secondary or higher & 71 & 157 & $0.56(0.39-0.81)$ & $0.86(0.46-1.62)$ & 0.650 \\
\hline \multicolumn{6}{|l|}{ Occupation ( $n=496$ ) } \\
\hline Farmer & 65 & 72 & 1 & 1 & \\
\hline Private employed & 34 & 55 & $0.68(0.39-1.18)$ & $1.91(0.68-5.32)$ & 0.215 \\
\hline Government & 34 & 67 & $0.56(0.33-0.96)$ & $1.14(0.39-3.31)$ & 0.805 \\
\hline Merchant/business & 72 & 85 & $0.94(0.59-1.48)$ & $1.67(0.70-3.95)$ & 0.242 \\
\hline Daily labor & 79 & 91 & $0.96(0.61-1.51)$ & $1.21(0.53-2.77)$ & 0.643 \\
\hline Student & 8 & 16 & $0.55(0.22-1.38)$ & $0.94(0.17-5.13)$ & 0.945 \\
\hline House wife & 18 & 51 & $0.39(0.21-0.74)$ & $0.59(0.23-1.52)$ & 0.279 \\
\hline Others* & 8 & 4 & $2.22(0.64-7.70)$ & $2.54(0.31-20.99)$ & 0.388 \\
\hline \multicolumn{6}{|l|}{ Wealth quintile status } \\
\hline Poorest & 82 & 86 & 1 & 1 & \\
\hline Poorer & 61 & 75 & $0.85(0.54-1.34)$ & $0.69(0.34-1.37)$ & 0.290 \\
\hline Middle & 58 & 91 & $0.67(0.42-1.04)$ & $0.62(0.29-1.28)$ & 0.195 \\
\hline Wealthier & 59 & 99 & $0.63(0.40-0.97)$ & $0.94(0.44-2.02)$ & 0.874 \\
\hline Wealthiest & 58 & 90 & $0.68(043-1.05)$ & $0.67(0.30-1.49)$ & 0.330 \\
\hline \multicolumn{6}{|l|}{ Zone } \\
\hline East Gojjam & 129 & 202 & $1.13(0.81-1.59)$ & $0.97(0.55-1.72)$ & 0.928 \\
\hline West Gojjam & 96 & 74 & $2.30(1.55-3.42)$ & $2.04(1.04-3.97)$ & 0.037 \\
\hline Bahir Dar city & 93 & 165 & 1 & 1 & \\
\hline \multicolumn{6}{|l|}{ Facility type } \\
\hline Health center & 222 & 156 & $4.22(3.10-5.75)$ & $3.06(1.90-4.92)$ & $<0.001$ \\
\hline Hospital & 96 & 285 & 1 & 1 & \\
\hline \multicolumn{6}{|c|}{ Residence of the patient } \\
\hline Rural & 89 & 93 & $1.45(1.04-2.03)$ & $1.84(0.94-3.60)$ & 0.075 \\
\hline Urban & 229 & 348 & 1 & 0 & \\
\hline \multicolumn{6}{|l|}{ HIV was diagnosed at } \\
\hline The same facility & 260 & 324 & $1.62(1.14-2.31)$ & $2.16(1.24-3.74)$ & 0.006 \\
\hline Another facility & 58 & 117 & 1 & 1 & \\
\hline \multicolumn{6}{|l|}{ Testing modality } \\
\hline Provider initiated & 199 & 314 & 1 & 1 & 0.782 \\
\hline Self-initiated & 119 & 127 & $1.48(1.09-2.01)$ & $0.93(0.56-1.52)$ & \\
\hline \multicolumn{6}{|c|}{ Life time sexual partner $(n=745)$} \\
\hline One & 141 & 164 & $1.31(0.98-1.76)$ & $1 . .41(0.87-2.27)$ & 0.162 \\
\hline more than one & 174 & 266 & 1 & 1 & \\
\hline \multicolumn{6}{|c|}{ Sexually active 3 months } \\
\hline Yes & 184 & 214 & $1.42(1.06-1.90)$ & $1.30(0.80-2.13)$ & 0.279 \\
\hline No & 131 & 216 & 1 & 1 & \\
\hline \multicolumn{6}{|c|}{ Ever use condom $(n=745)$} \\
\hline Yes & 65 & 132 & 1 & 1 & \\
\hline No & 250 & 298 & $1.70(1.21-2.40)$ & $1.61(0.87-3.00)$ & 0.131 \\
\hline
\end{tabular}


Table 5 Factors associated with uptake of same-day ART initiation in northwest Ethiopia, 2019 (Continued)

\begin{tabular}{|c|c|c|c|c|c|}
\hline Characteristics & Same-day initiated & Not initiated & COR $95 \% \mathrm{Cl}$ & AOR $(95 \% \mathrm{Cl})$ & $P$-value \\
\hline \multicolumn{6}{|c|}{ Sex out of regular partner $(n=745)$} \\
\hline Yes & 59 & 102 & 1 & 1 & \\
\hline No & 256 & 325 & $1.35(0.94-1.93)$ & $0.76(0.39-1.49)$ & 0.425 \\
\hline \multicolumn{6}{|l|}{ Ever use alcohol } \\
\hline Yes & 215 & 331 & 1 & 1 & \\
\hline No & 103 & 110 & $1.44(1.04-1.98)$ & $1.45(0.90-2.34)$ & 0.126 \\
\hline \multicolumn{6}{|l|}{ Opportunistic infection } \\
\hline Yes & 65 & 144 & 1 & 1 & \\
\hline No & 253 & 297 & $1.89(1.35-2.64)$ & $2.08(1.04-4.19)$ & 0.039 \\
\hline \multicolumn{6}{|l|}{ WHO clinical stage } \\
\hline$|/| \mid$ & 264 & 305 & $2.18(1.53-3.11)$ & $0.80(0.38-1.64)$ & 0.542 \\
\hline III/IV & 54 & 136 & 1 & 1 & \\
\hline \multicolumn{6}{|c|}{ Pregnancy status ( $n=436$ ) } \\
\hline Pregnant & 31 & 15 & $3.20(1.67-6.13)$ & $3.97(1.78-8.87)$ & 0.001 \\
\hline Not pregnant & 153 & 237 & 1 & 1 & \\
\hline \multicolumn{6}{|l|}{ Living arrangement } \\
\hline Living with someone & 211 & 273 & $1.21(0.89-1.64)$ & $1.36(0.83-2.21)$ & 0.220 \\
\hline Living alone & 107 & 168 & 1 & & \\
\hline \multicolumn{6}{|c|}{ HIV prevention knowledge } \\
\hline Good & 198 & 318 & 1 & 1 & \\
\hline Poor & 120 & 123 & $1.57(1.15-2.13)$ & $1.10(0.67-1.79)$ & 0.696 \\
\hline \multicolumn{6}{|l|}{ Perceived stigma } \\
\hline High & 262 & 337 & $1.44(1.01-2.07)$ & $1.23(0.68-2.22)$ & 0.488 \\
\hline Low & 56 & 104 & 1 & 1 & \\
\hline
\end{tabular}

give priority to treat acute opportunistic infection due to fear of pill burden rather than initiating ART. This approach causes delay in treatment initiation [1]. And explains why patients who presented with TB as an opportunistic infection were mostly not initiated on sameday ART treatment $(P<0.002)$. The limitation of the current study is in addition to lack of baseline CD4 determination, it included only the same-day ART initiation and did not follow how long they delayed and did not study the healthcare providers' behavior that affects timely ART initiation. Hence, it is better to determine the baseline CD4 count to ascertain the advancement of disease. Follow-up of those patients who differed rapid ART initiation for a reasonable time is necessary to document barriers of treatment initiation in the course of time.

Uptake of same-day ART among pregnant women was $67.4 \%$ a value lower that the report from a study in Zimbabwe in which over $80 \%$ of those who underwent HIV testing in maternal and child health departments initiated ART on the same-day [55]. Similarly, two different studies in Cape Town South Africa reported that $73 \%$ of pregnant women initiated ART same-day [30] and $91 \%$ of women initiated onto ART starting the same day treatment eligibility was determined [27]. However, the present finding was better than a study in Malawi (51 and 63\%) [31] depending on service integration with HIV clinic and ANC. The difference can be attributed due to differences in socio-economic status of the patients, the study design and the study period. Most importantly, pregnant women were four times more likely to initiate same-day ART compared to the general adult HIV positive population. The reason for better acceptance of same-day ART initiation among pregnant women can be explained by the emphasis given by both WHO and the Ethiopian government to initiate immediate ART for pregnant and lactating women $[1,2]$. Moreover, pregnant and lactating women are ready to accept same-day ART due to their commitment to their child's health [25].

Newly diagnosed HIV positive patients in a health facility in which they were linked to ART service were two times more likely to initiate same-day ART compared with those tested HIV positive from another health facility and subsequently referred. This was due to the time needed to transfer from the original HIV testing site to 
the actual health facility rendering ART service. A significant number of our study participants knew their HIV status in private and non-governmental health facilities. Similarly, in Tanzania, HIV testing in the community setting was a reason for delayed treatment initiation [24]. This emphasizes the need for active referral mechanism between HIV testing sites and ART clinics to shorten the time between HIV diagnosis and ART initiation. Well established referral systems can increase uptake of ART [57].

New HIV patients who sought HIV treatment and support services at health centers were three times more likely to initiate same-day ART compared to those at hospitals. This finding is supported by previous studies in Ethiopia that concluded, HIV treatment in health centers was feasible and as effective as in hospitals [59-63]. Additionally, hospitals are overloaded with many patients that may cause lack of time for adequate patient preparation for ART initiation within the same-day of HIV diagnosis. Furthermore, patients treated in West Gojjam were two times more likely to initiate ART compared to the regional state capital-Bahir Dar city administration. The possible reason can be that health facilities in cities like Bahir Dar are overloaded. In addition to busy clinics, urban residents and educated people were less likely to accept ART on the days of HIV diagnosis. The implications of the above findings is in addition to patient and clinician factors affecting same-day treatment initiation, the health system management at the zonal level may also affect the program's effectiveness at large.

\section{Limitations of the study}

Social desirability bias may influence this study due to persistent natures of HIV related stigma in the community. Inclusion of TB patients in this study may underestimated the magnitude of SDI though there were few patients who initiated same-day ART. Some patients were not stable at the time of their HIV diagnosis and we postponed data collection to the next visit within a week. As a result, some of them did not come back to the clinic and this may have introduced bias by avoiding most at-risk patients for delayed ART initiation. As a result, the finding may not be generalized for such patients. Additionally, this study investigated factors only from the patient side characteristics and may not explain the healthcare providers' perspective on the same-day ART initiation. Therefore, further study is recommended to uncover the role of healthcare workers in same-day ART initiation.

\section{Conclusion}

Less than half of newly diagnosed HIV positive people initiated ART in the same-day of HIV diagnosis in
Ethiopia. None of the socio-demographic, behavioral and psychological factors were associated with differing of same-day ART initiation. Rather, clinical factors such as having opportunistic infections and non-pregnancy status affected the immediate initiation of treatment. Others like being in a hospital and major city-Bahir Dar were also factors. This indicated that same-day ART initiation was affected by healthcare providers' decision and organizational factors like patient load and the strength of health system management.

\section{Abbreviations \\ HAART: Highly Active Antiretroviral Therapy; HIV: Human immunodeficiency virus; PLHIV: People Ling with HIV; WHO: World Health Organization}

\section{Acknowledgments}

We are grateful to African Union Commission (AU) for funding this study. We also extend our gratitude to our research assistants all study participants who participated in this study.

\section{Authors' contributions}

NAM, OAA, MAO and YB contributed equally to the design of the study. NAM and OAA drafted the manuscript and all the authors revised and approved the manuscript.

\section{Funding}

This study was supported by the Pan African University (PAU), a continental initiative of the African Union Commission (AU), Addis Ababa, Ethiopia, as part of the Ph.D. program in Reproductive Health Sciences. NAM received the funding from PAU. The University had no role in the study design and decision to publish, or preparation of the manuscript.

\section{Availability of data and materials}

The datasets used and/or analyzed during the current study are available from the corresponding author on reasonable request.

\section{Ethics approval and consent to participate}

Institutional review board (IRB) of University College Hospital, University of Ibadan, Ibadan, Nigeria on 17/09/2018 with reference number UI/EC/18/0463 and Debre Markos University with reference number HSC/30/02/2011 have approved.

\section{Consent for publication}

Not applicable.

\section{Competing interests}

The authors declared that there is no competing of interest.

\section{Author details}

'Department of Public Health, College of Health Sciences, Debre Markos University, Debre Markos, Ethiopia. ${ }^{2}$ Pan African University, Life and Earth Sciences Including Health and Agriculture Institute (PAULESI), University of Ibadan, Ibadan, Nigeria. ${ }^{3}$ Department of Obstetrics and Gynecology, College of medicine, University of Ibadan, Ibadan, Nigeria. ${ }^{4}$ Department of Obstetrics and Gynecology, University College Hospital, Ibadan, Nigeria. ${ }^{5}$ Department of Epidemiology, Addis Continental Institute of Public Health, Addis Ababa, Ethiopia.

Received: 13 April 2020 Accepted: 15 September 2020

Published online: 22 September 2020

\footnotetext{
References

1. WHO, Guidelines for managing advanced HIV disease and rapid initiation of antiretroviral therapy, July 2017. 2017.

2. Ethiopia, F.M.o.H. In: Health, editor. National guidelines for comprehensive HIV prevention, care and treatment, A.A.M.o; 2017.

3. Boyd M, et al. Rapid initiation of antiretroviral therapy at HIV diagnosis: definition, process, knowledge gaps. HIV Med. 2019;20:3-11.
} 
4. Patel, R.R., K.A. Curoe, And P.A. Chan, Undetectable Equals Untransmittable: A Game Changer for HIV Prevention. Clin Chem, 2020.

5. Thornhill J, et al. The East London Immediate ART (ELIA) survey-Attitudes \& Barriers to Immediate ART initiation. in HIV MEDICINE. NJ USA: WILEY 111 RIVER ST, HOBOKEN 07030-5774; 2019.

6. Rodger AJ, et al. Risk of HIV transmission through condomless sex in serodifferent gay couples with the HIV-positive PARTNER taking suppressive antiretroviral therapy (PARTNER): final results of a multicentre, prospective, observational study. Lancet. 2019;393(10189):2428-38.

7. Mateo-Urdiales A, et al. Rapid initiation of antiretroviral therapy for people living with HIV. Cochrane Database Syst Rev. 2019;6.

8. Huang $Y$-C, et al. Short-term outcomes of rapid initiation of antiretroviral therapy among HIV-positive patients: real-world experience from a singleCentre retrospective cohort in Taiwan. BMJ Open. 2019;9(9):e033246.

9. Bacon, O., et al. The Rapid ART Program Initiative for HIV Diagnoses (RAPID) in San Francisco. in 25th Conference on Retroviruses and Opportunistic Infections (CROI). 2018.

10. Zhao $Y$, et al. Immediate antiretroviral therapy decreases mortality among patients with high CD4 counts in China: a nationwide, retrospective cohort study. Clin Infect Dis. 2017.

11. Lee MJ, et al. Reasons for delayed antiretroviral therapy (ART) initiation in the era of early ART initiation guidelines: a retrospective service evaluation. Int J STD AIDS. 2019;30(4):415-8.

12. Kebaabetswe $P$, et al. Barriers and facilitators to linkage to care and ART initiation in the setting of high ART coverage in Botswana. AIDS Care. 2019: $1-7$.

13. Kiwanuka J, et al. Determinants of loss to follow-up among HIV positive patients receiving antiretroviral therapy in a test and treat setting: a retrospective cohort study in Masaka, Uganda. PLoS One. 2020;15(4): e0217606.

14. Stafford KA, et al. Evaluation of the clinical outcomes of the test and treat strategy to implement treat all in Nigeria: results from the Nigeria multiCenter ART study. PLoS One. 2019;14(7):e0218555.

15. Lilian RR, et al. Same-day antiretroviral therapy initiation for HIV-infected adults in South Africa: analysis of routine data. PLoS One. 2020;15(1): e0227572.

16. Govindasamy D, Ford N, Kranzer K. Risk factors, barriers and facilitators for linkage to antiretroviral therapy care: a systematic review. Aids. 2012;26(16): 2059-67.

17. Croxford S, et al. Linkage to HIV care following diagnosis in the WHO European region: a systematic review and meta-analysis, 2006-2017. PLoS One. 2018;13(2):e0192403.

18. Layer $\mathrm{EH}$, et al. Multi-level factors affecting entry into and engagement in the HIV continuum of Care in Iringa, Tanzani. PLoS One. 2014;9(8):e104961.

19. Ford N, et al. Benefits and risks of rapid initiation of antiretroviral therapy. AIDS (London, England). 2018;32(1):17.

20. Boyer $\mathrm{S}$, et al. Factors associated with antiretroviral treatment initiation amongst HIV-positive individuals linked to care within a universal test and treat programme: early findings of the ANRS 12249 TasP trial in rural South Africa. AIDS Care. 2016;28(sup3):39-51.

21. Gesesew HA, et al. Late presentation for HIV care in Southwest Ethiopia in 2003-2015: prevalence, trend, outcomes and risk factors. BMC Infect Dis. 2018;18(1):59.

22. Labhardt ND, et al. Same day ART initiation versus clinic-based pre-ART assessment and counselling for individuals newly tested HIV-positive during community-based HIV testing in rural Lesotho-a randomized controlled trial (CASCADE trial). BMC Public Health. 2016;16(1):329.

23. Hoenigl M, et al. Rapid HIV viral load suppression in those initiating antiretroviral therapy at first visit after HIV diagnosis. Sci Rep. 2016;6:32947.

24. Reddy EA, et al. Test site predicts HIV care linkage and antiretroviral therapy initiation: a prospective 3.5 year cohort study of HIV-positive testers in northern Tanzania. BMC Infect Dis. 2016;16(1):497.

25. Hodgson I, et al. A systematic review of individual and contextual factors affecting ART initiation, adherence, and retention for HIV-infected pregnant and postpartum women. PLoS One. 2014;9(11):e111421.

26. Mbonye $\mathrm{M}$, et al. Test and treat: the early experiences in a clinic serving women at high risk of HIV infection in Kampala. AIDS Care. 2016;28(sup3): 33-8.

27. Samantha Black RZ, Marcus R, Mark D, Myer L, Bekker L-G. Acceptability and challenges of rapid ART initiation among pregnant women in a pilot programme, Cape Town, South Africa. AIDS Care. 2014;26(6):736-41.
28. Katz IT, et al. Understanding treatment refusal among adults presenting for HIV-testing in Soweto, South Africa: a qualitative study. AIDS Behav. 2015; 19(4):704-14.

29. Assefa $Y$, et al. Toward universal access to HIV counseling and testing and antiretroviral treatment in Ethiopia: looking beyond HIV testing and ART initiation. AIDS Patient Care STDs. 2010;24(8):521-5.

30. Langwenya N, et al. Same-day antiretroviral therapy (ART) initiation in pregnancy is not associated with viral suppression or engagement in care: a cohort study. J Int AIDS Soc. 2018;21(6):e25133.

31. Chan AK, et al. Same day HIV diagnosis and antiretroviral therapy initiation affects retention in option B+ prevention of mother-to-child transmission services at antenatal care in Zomba District, Malawi. J Int AIDS Soc. 2016;19(1):20672.

32. Koenig SP, et al. Same-day HIV testing with initiation of antiretroviral therapy versus standard care for persons living with HIV: a randomized unblinded trial. PLoS Med. 2017;14(7):e1002357.

33. Grinsztejn B, et al. Effects of early versus delayed initiation of antiretroviral treatment on clinical outcomes of HIV-1 infection: results from the phase 3 HPTN 052 randomised controlled trial. Lancet Infect Dis. 2014;14(4):281-90.

34. Horter $\mathrm{S}$, et al. "Is it making any difference?" A qualitative study examining the treatment-taking experiences of asymptomatic people living with HIV in the context of Treat-all in Eswatini. J Int AIDS Soc. 2019;22(1):e25220.

35. Kiriazova T, O. P, Bingham T, Myers J, Flanigan T, Vitek C, Neduzhko AO. Patient and provider perspectives inform an intervention to improve linkage to care for HIV patients in Ukraine. BMC Health Serv Res. 2018.

36. Ayieko J, et al. "Hurdles on the path to 90-90-90 and beyond": Qualitative analysis of barriers to engagement in HIV care among individuals in rural East Africa in the context of test-and-treat. PLoS One. 2018;13(8):e0202990.

37. Horter $\mathrm{S}$, et al. "Life is so easy on ART, once you accept it": acceptance, denial and linkage to HIV care in Shiselweni, , Swaziland. Soc Sci Med. 2017; 176:52-9.

38. Adams AK, Zamberia AM. "I will take ARVs once my body deteriorates": an analysis of Swazi men's perceptions and acceptability of Test and Start. Afr J AIDS Res. 2017;16(4)

39. Kuznetsova AV, et al. Barriers and facilitators of HIV care engagement: results of a qualitative study in St. Petersburg, Russia. AIDS Behav. 2016;20(10): 2433-43.

40. MacPherson $P$, et al. Barriers and facilitators to linkage to ART in primary care: a qualitative study of patients and providers in Blantyre, Malawi. J Int AIDS Soc. 2012;15(2):18020.

41. Teklu AM, et al. Exploratory analysis of time from HIV diagnosis to ART start, factors and effect on survival: a longitudinal follow up study at seven teaching hospitals in Ethiopia. Ethiop J Health Sci. 2017;27(1):17-28.

42. Gudina EK, et al. Magnitude of antiretroviral drug toxicity in adult HIV patients in Ethiopia: a cohort study at seven teaching hospitals. Ethiop J Health Sci. 2017;27(1):39-52.

43. Nash D, et al. Factors associated with initiation of antiretroviral therapy in the advanced stages of HIV infection in six Ethiopian HIV clinics, 2012 to 2013. J Int AIDS Soc. 2016;19(1)

44. Aniley $A B$, et al. Factors associated with late human immunodeficiency virus (HIV) diagnosis among peoples living with it, Northwest Ethiopia: hospital based unmatched case-control study. BMC Public Health. 2016;16(1):1076.

45. Gelaw YA, et al. Determinants of late presentation to HIV/AIDS care in southern Tigray zone, northern Ethiopia: an institution based case-control study. AIDS Res Ther. 2015;12(1):40.

46. Gesesew HA, Tesfamichael FA, Adamu BT. Factors affecting late presentation for HIV/AIDS care in Southwest Ethiopia: a case control study. Public Health Res. 2013;3(4):98-107.

47. CSA, I., Census 2007 Tables: Amhara Region Archived November 14, 2010, at the Wayback Machine. 2007: online accessed on June 30, 2018.

48. Sabapathy $K$, et al. Predictors of timely linkage-to-ART within universal test and treat in the HPTN 071 (PopART) trial in Zambia and South Africa: findings from a nested case-control study. J Int AIDS Soc. 2017;20(4):e25037.

49. Tsadik M, et al. The magnitude of, and factors associated with, loss to follow-up among patients treated for sexually transmitted infections: a multilevel analysis. BMJ Open. 2017;7(7):e016864.

50. Kulkarni SP, et al. Clinical uncertainties, health service challenges, and ethical complexities of HIV "test-and-treat": a systematic review. Am J Public Health. 2013;103(6):e14-23.

51. Group, S.f.M.o.A.T.S. Major clinical outcomes in antiretroviral therapy (ART)naive participants and in those not receiving ART at baseline in the SMART study. J Infect Dis. 2008;197(8):1133-44. 
52. Ankomah A, et al. ART access-related barriers faced by HIV-positive persons linked to care in southern Ghana: a mixed method study. BMC Infect Dis. 2016;16(1):738

53. Lifson $A R$, et al. Barriers to retention in care as perceived by persons living with HIV in rural Ethiopia: focus group results and recommended strategies. J Int Assoc Providers AIDS Care (JIAPAC). 2013;12(1):32-8

54. Kessler. Kessler Psychological Distress Scale (K10). Boston: Harvard Medical School; 2001. online accessed on July 2, 2018.

55. Rufu A, et al. Implementation of the 'test and Treat'policy for newly diagnosed people living with HIV in Zimbabwe in 2017. Public Health Action. 2018;8(3):145-50.

56. Pilcher $C D$, et al. The effect of same-day observed initiation of antiretroviral therapy on HIV viral load and treatment outcomes in a US public health setting. J Acquir Immune Defic Syndr (1999). 2017;74(1):44.

57. Coffey S, et al. RAPID antiretroviral therapy: high virologic suppression rates with immediate antiretroviral therapy initiation in a vulnerable urban clinic population. Aids. 2019;33(5):825-32.

58. Nhassengo $P$, et al. Barriers and facilitators to the uptake of test and treat in Mozambique: a qualitative study on patient and provider perceptions. PLoS One. 2018;13(12):e0205919.

59. Reepalu A, et al. High rates of virological suppression in a cohort of human immunodeficiency virus-positive adults receiving antiretroviral therapy in ethiopian health centers irrespective of concomitant tuberculosis. In: Open forum infectious diseases: Oxford University Press; 2014.

60. Mitiku I, et al. Factors associated with loss to follow-up among women in option B+ PMTCT programme in Northeast Ethiopia: a retrospective cohort study. J Int AIDS Soc. 2016;19(1):20662.

61. Teshome W, et al. Do loss to follow-up and death rates from ART care vary across primary health care facilities and hospitals in south Ethiopia? A retrospective follow-up study. HIV/AIDS (Auckland, NZ). 2015;7:167.

62. Bucciardini $\mathrm{R}$, et al. Retention in care of adult HIV patients initiating antiretroviral therapy in Tigray, Ethiopia: a prospective observational cohort study. PLoS One. 2015;10(9):e0136117.

63. Balcha TT, Jeppsson A. Outcomes of antiretroviral treatment: a comparison between hospitals and health centers in Ethiopia. J Int Assoc Phys AIDS Care. 2010;9(5):318-24.

\section{Publisher's Note}

Springer Nature remains neutral with regard to jurisdictional claims in published maps and institutional affiliations.

Ready to submit your research? Choose BMC and benefit from:

- fast, convenient online submission

- thorough peer review by experienced researchers in your field

- rapid publication on acceptance

- support for research data, including large and complex data types

- gold Open Access which fosters wider collaboration and increased citations

- maximum visibility for your research: over $100 \mathrm{M}$ website views per year

At BMC, research is always in progress.

Learn more biomedcentral.com/submissions 